\title{
Improved Poly (D,L-lactide) nanoparticles-based formulation for hair follicle targeting
}

\author{
B. Fernandes*, R. Silva*, A. Ribeiro ${ }^{*, \dagger}$, T. Matamá ${ }^{* \dagger}$, A. C. Gomes ${ }^{\dagger}$ and A. M. Cavaco-Paulo* \\ ${ }^{*}$ Centre of Biological Engineering (CEB), University of Minho, Campus of Gualtar, 4710-057 Braga, and ${ }^{\dagger}$ Centre of Molecular and Environmental \\ Biology (CBMA), University of Minho, Campus of Gualtar, 4710-057 Braga, Portugal
}

Received 9 October 2014, Accepted 25 December 2014

Keywords: delivery/vectorization/penetration, formulation/stability, hair follicles, hair treatment, nanoprecipitation, poly (D,L-lactide)

\begin{abstract}
Synopsis
OBJECTIVE: Hair follicles are widely recognized as the preferential target and site of accumulation for nanoparticles after topical application. This feature is of particular importance for hair cosmetics, having the potential to refine the treatment of several hair folliclerelated disorders. The aim of this work was to improve the preparation of Poly (D,L-lactide) (PLA) nanoparticles for in vivo follicular target and drug delivery.

METHODS: Envisaging a future industrial scale-up of the process, nanoprecipitation method was used to prepare PLA nanoparticles: the effect of several processing parameters on their properties was examined and the yield of nanoparticles formation determined. Encapsulation efficiencies and in vitro release profiles of lipophilic and hydrophilic model compounds were also assessed. In vitro cytotoxicity and ex vivo penetration studies were performed on a reference skin cell line (NCTC2455, human skin keratinocytes) and porcine skin, respectively.

RESULTS: Using acetone : ethanol $(50: 50, \mathrm{v} / \mathrm{v})$ as the solvent phase, $0.6 \%(\mathrm{w} / \mathrm{w})$ of Pluronic ${ }^{\circledR} \mathrm{F} 68$ as a surfactant agent and agitation to mix the solvent and non-solvent phases, a monodispersed population of non-cytotoxic spherical nanoparticles of approximately $150 \mathrm{~nm}$ was obtained. The yield of nanoparticles for this formulation was roughly $90 \%$. After encapsulation of model compounds, no significant changes were found in the properties of particles and the entrapment efficiencies were above $80 \%$. The release kinetics of dyes from PLA nanoparticles indicate an anomalous transport mechanism (diffusion and polymer degradation) for Nile Red (lipophilic) and a Fickian diffusion of first order for fluorescein 5(6)-isothiocyanate (hydrophilic). Ex vivo skin penetration studies confirmed the presence of nanoparticles along the entire follicular ducts.

CONCLUSIONS: The optimized method allows the preparation of ideal PLA nanoparticles-based formulations for hair follicle targeting. PLA nanoparticles can effectively transport and release lipophilic and hydrophilic compounds into the hair follicles, and the yields obtained are acceptable for industrial purposes.
\end{abstract}

\section{Résumé}

OBJECTIF: Les follicules pileux sont largement reconnus comme la cible préférentielle et le site de l'accumulation des nanoparticules

Correspondence: Artur Cavaco-Paulo, Centro de Engenharia Biológica (CEB), Universidade do Minho, 4710-057 Braga, Portugal.

Tel.: +351 253604 409; fax: +351253604 429;

e-mail: artur@deb.uminho.pt après application topique. Cette caractéristique est particulièrement importante pour les produits cosmétiques pour les cheveux, ayant la possibilité d'affiner le traitement de plusieurs troubles des follicules de cheveux. Le but de ce travail était d'améliorer la préparation de nanoparticules poly (D,L-lactide) (PLA) pour une administration folliculaire in vivo ciblée de drogues.

MÉTHODES: En envisageant un avenir à l'échelle industrielle du procédé, une méthode de nanoprécipitation a été utilisé pour préparer des nanoparticules de PLA: l'effet de plusieurs paramètres de traitement sur leurs propriétés a été examiné et le rendement de la formation des nanoparticules a été déterminé. Les efficacités d'encapsulation et de profils de libération in vitro de composés modèles lipophiles et hydrophiles ont également été évaluées. La cytotoxicité in vitro et ex vivo des études de pénétration a été effectuée sur une lignée de cellules de peau de référence (NCTC2455, des kératinocytes de peau humaine) et la peau de porc, respectivement. RESULTATS: En utilisant l'acétone : éthanol (50:50, v/v) comme phase solvant, $0,6 \%(\mathrm{p} / \mathrm{p})$ de Pluronic ${ }^{\circledR} \mathrm{F} 68$ à titre d'agent tensioactif et l'agitation pour mélanger les phases de solvant et de non-solvant, une population monodispersée des nanoparticules sphériques non cytotoxiques d'environ $150 \mathrm{~nm}$ a été obtenue. Le rendement de nanoparticules pour cette formulation était d'environ $90 \%$. Après encapsulation de composés modèles, aucune modification significative n'a été observée dans les propriétés des particules et les efficacités de piégeage ont été supérieures à $80 \%$. La cinétique de libération de colorants de nanoparticules de PLA indique un mécanisme de transport anormal (diffusion et dégradation de polymère) pour le rouge Nil (lipophile) et une diffusion selon Fick de premier ordre pour FITC (hydrophile). Les études de pénétration $e x$ vivo de la peau ont confirmé la présence de nanoparticules sur tous les conduits folliculaires.

CONCLUSIONS: La méthode optimisée permet la préparation de formulations à base de nanoparticules de PLA, idéales pour ciblage $\mathrm{du}$ follicule pileux. Les nanoparticules de PLA peuvent effectivement transporter et libérer des composés lipophiles et hydrophiles dans les follicules pileux; les rendements obtenus sont acceptables à des fins industrielles.

\section{Introduction}

Nanoparticles are seen today as a key tool for the development of new cosmetics products: they improve the stability of various active ingredients, enhance their penetration into the skin and make some products more aesthetically pleasing [1]. 
Hair follicles are the preferential entrance point of nanoparticles into the skin [2]. The penetration within the follicular duct is dependent on their diameter, with smaller nanoparticles showing increased penetration depths [3]. Concomitantly, if nanoparticles are larger than $100 \mathrm{~nm}$, they are not able to translocate into the viable epidermis, being trapped inside these structures [4]. This localized accumulation is especially important for the treatment of hair follicle-associated diseases (e.g. acne) and has the potential to revolutionize the treatment of human hair disorders (e.g. hirsutism, greying and alopecia) that have dramatic effects on the appearance, sociocultural status and self-esteem of the affected individuals [4-6]. As the drugs entrapped inside nanoparticles will be released just at the specific site of action, their bioavailability is enhanced. Consequently, minimal concentrations of active substances can be administered to achieve a desired effect, enabling the utilization of many pharmacological compounds for follicular therapy that otherwise would be unfeasible $[3,7,8]$.

Polymeric nanoparticles are the most promising technology for follicular target as they exhibit great stability and efficiently mask the intrinsic properties of encapsulated drugs. These features facilitate the entrance of therapeutic agents that have low solubility on the sebum found inside the hair follicles [9]. Currently, nanoprecipitation (first introduced by Fessi et al.) is the most commonly used technique to formulate polymeric nanoparticles intended for topical application. In this method, the polymer is dissolved in an organic phase and the solution obtained is added to an aqueous phase (wherewith it is miscible). As the organic solvent flows away and diffuses in the aqueous phase, polymer molecules aggregate (due to the loss of solubility) and precipitate in the form of nanoparticles. Once the organic solvent is removed, the particles harden. Although it suffers the drawback of poor encapsulation efficiency of water-soluble drugs, nanoprecipitation allows the preparation of small nanoparticles with narrow size distribution in a simple, fast, economic and highly reproducible manner $[9,10]$.

In a recent study, Rancan et al. [4] proved the ability of Poly (D, L-lactide) nanoparticles (228 and $365 \mathrm{~nm}$ ) prepared with the original nanoprecipitation method to efficiently target the hair follicles, reaching a maximal depth corresponding to the entry of sebaceous gland. In this work, we aimed to optimize this protocol in order to obtain smaller PLA nanoparticles. These nanoparticles will be able to achieve a deeper penetration into the hair follicles, allowing the delivery of drugs at the level of the hair bulb. The bulb of hair follicles is one of the most interesting targets for hair cosmetics due to the presence of hair matrix cells (that play an important role in the control of hair growth) and melanocytes (involved in the production of melanin and consequent coloration of hair shafts) $[3,6$, $11]$.

The physical properties (and especially size) of nanoparticles obtained with nanoprecipitation are known to be dependent on many processing parameters: polymer, solvent, nature of the polymer-solvent interactions and non-solvent system [12]. Thus, the influence of the composition of the solvent phase, concentration of surfactant and technique used to mix both phase was studied. Once established the optimal conditions for the preparation of PLA nanoparticles, the entrapment efficiencies and release profiles of hydrophilic and lipophilic model compounds were determined. $E x$ vivo assays on porcine skin were performed to confirm the distribution of nanoparticles on the skin. Although the utilization of PLA is related to the fact that it is biocompatible, biodegradable and already approved by Food and Drug Administration (FDA) for clinic use, the cytotoxicity of nanoparticles on a reference skin line was determined [13].

\section{Materials and methods}

\section{Chemicals and solvents}

Poly (D,L-lactide) $(\mathrm{Mw}=18-24 \mathrm{kDa})$, Pluronic ${ }^{\circledR}$ F68 (suitable for cell culture), 3-[4,5-dimethylthiazol-2-yl]-2,5-diphenyl tetrazolium bromide (MTT), methanol (ACS reagent grade, $\geq 99.8 \%$ ), nile red (suitable for fluorescence), fluorescein 5(6)-isothiocyanate (suitable for fluorescence) and isopropyl myristate (Kosher grade, $\geq 98 \%$ ) were purchased from Sigma-Aldrich (Sigma Chemical Co., St. Louis, MO, U.S.A.). Acetone, ethanol and dimethyl sulphoxide (DMSO) were of the highest grade commercially available and purchase from several suppliers.

\section{Preparation of PLA nanoparticles}

PLA nanoparticles were prepared based on the nanoprecipitation method [10]. Briefly, the polymer was dissolved in acetone $-2 \%$ $(\mathrm{w} / \mathrm{w})$ - and added to an aqueous phase containing Pluronic ${ }^{\circledR}$ F68 (solvent phase to aqueous phase ratio was $1: 2$ ). The concentration of surfactant in the resultant solution was $0.6 \%(\mathrm{w} / \mathrm{w})$. After formation of nanoparticles, the organic phase was completely removed with a rotary evaporator. The aqueous suspensions of nanoparticle were stored at $4^{\circ} \mathrm{C}$.

The cumulative effect of various processing parameters on the properties of PLA nanoparticles was studied. Three different techniques (agitation, sonication and homogenization) were used to mix the solvent and aqueous phases. Agitation: the organic phase was added dropwise into the aqueous phase under magnetic stirring at 100 r.p.m. The solution obtained was stirred overnight at the same velocity. Sonication: organic phase was added to the aqueous phase and the preparation submitted to an ultrasound treatment (power delivery controlled as percentage amplitude at $40 \%$, $18 \mathrm{~min}, 5 \pm 1^{\circ} \mathrm{C}$ ) [14]. The experimental set-up used was composed of an ultrasound probe (20 kHz Vibracellтм CV 33) fitted with a 3-mm diameter titanium micro-tip (Sonics and Materials Inc., Newtown, CT, U.S.A.). Homogenization: after addition of the solvent phase to the aqueous phase, the solution was homogenized

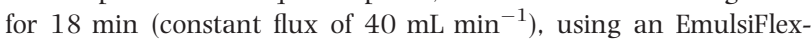
C3 (Avestin, Ottawa, ON, Canada). PLA nanoparticles were also prepared using a fraction of a non-solvent (ethanol or water) in the solvent phase. Using titration, $55 \%$ and $10 \%(\mathrm{v} / \mathrm{v})$ were determined as the maximum proportions of ethanol and water for the complete dissolution of the polymer. Finally, the effect of the concentration of Pluronic ${ }^{\circledR}$ F68 was studied.

\section{Experimental yield of nanoparticles}

The suspensions of nanoparticles were filtered to remove polymeric aggregates above the range of nano sizes. All the materials that contained the formulations were washed with acetone to dissolve the attached polymer. Methanol was added to precipitate PLA, and the solvents were removed on rotary evaporator. The polymer recovered in the filter and from the materials was dried and weighed (mass of aggregates, $m_{\text {agg }}$ ). The yield of nanoparticles was calculated as weight percent in relation to the initial amount of polymer used in formulation $\left(m_{\text {total }}\right)[12]$ : 


$$
\text { Yield of Nanoparticles }(\%)=\frac{m_{\mathrm{total}}-m_{\mathrm{agg}}}{m_{\mathrm{total}}} \times 100 \text {. }
$$

\section{Particle size, size distribution and zeta-potential}

The mean size and polydispersity (index from 0.0 to 1.0) of nanoparticles were assessed by Photon Correlation Spectroscopy (PCS), whereas laser Doppler anemometry (LDA) was employed for zeta

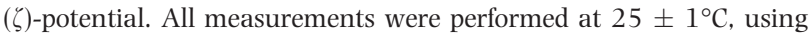
a ZetaSizer Nano ZS equipment (Malvern Instruments Ltd, Worcestershire, U.K.). The physical stability of suspensions was evaluated over 6 months, by weekly measurements of size, polydispersity index (PDI) and $\zeta$-potential of PLA nanoparticles stored at $4^{\circ} \mathrm{C}$.

\section{Shape and surface morphology}

The shape and surface morphology of nanoparticles were analysed by scanning transmission electron microscopy (STEM). The solutions containing nanoparticles were diluted and placed onto copper grids with carbon film 3-mm 400 meshes. Then, the observations were performed at $15 \mathrm{kV}$, using a NOVA Nano SEM 200 (FEITM, Hillsboro, OR, U.S.A.).

\section{Entrapment and loading efficiencies}

Dye-loaded nanoparticles were obtained employing the optimized nanoprecipitation protocol, with $1 \%(\mathrm{w} / \mathrm{v})$ of nile red $(\mathrm{NR})$ or fluorescein 5(6)-isothiocyanate (FITC) being dissolved along with the polymer in the organic phase. NR and FITC were used as models of lipophilic and hydrophilic compounds, respectively. The aqueous suspensions of nanoparticles were dialysed against distilled water (Mw cut-off of $12.4 \mathrm{kDa}$ ) to remove free dye in the formulation. The suspensions were freeze-dried, nanoparticles powder obtained weighed and dissolved in acetone. The encapsulated drug was measured by Ultraviolet (UV)-Spectrophotometry. For Nile Red, measurements were performed with an excitation wavelength of $536 \mathrm{~nm}$ and emission wavelength of $608 \mathrm{~nm}$. For FITC, 495 and $525 \mathrm{~nm}$ were used as excitation and emission wavelengths, respectively. The entrapment and loading efficiencies were calculated according to the following equations:

$$
\begin{gathered}
\text { Entrapment Efficiency }(\%)=\frac{m_{\text {encapsulated dye }}}{m_{\text {dye used in formulation }}} \times 100, \\
\text { Loading Efficiency }(\%)=\frac{m_{\text {encapsulated dye }}}{m_{\text {nanoparticles }}} \times 100 .
\end{gathered}
$$

\section{In vitro release profiling}

Aqueous suspensions of dye-loaded nanoparticles were added to vials containing the same volume of isopropyl myristate (IPM). The chemical structure of IPM resembles that of sebum components, and consequently, it can simulate the skin surface/interior of the hair follicle ducts [4]. The systems were constantly stirred at $37^{\circ} \mathrm{C}$. To assess the release profile of $\mathrm{NR}$, at fixed time points $(0,2,4,6$, 8,12 and $24 \mathrm{~h}$ ), a small aliquot of the lipophilic phase was collected and replaced with the same volume of IPM; fluorescence intensity was measured at $570 \mathrm{~nm}$ (excitation: $520 \mathrm{~nm}$ ) [15]. For FITC-loaded nanoparticles, at the same time points, the hydrophilic phase was collected, centrifuged to deposit nanoparticles and supernatant fluorescence measured at $525 \mathrm{~nm}$ (excitation at $495 \mathrm{~nm}$ ). The supernatant was discharged, nanoparticles were dispersed in water and added again to the lipophilic phase [16]. The amount of released dyes was determined using a calibration curve, obtained from the values of fluorescence intensity of standard samples. The percentages were calculated with respect to the initial amount present in the nanoparticles.

With the release rates of fluorochromes, it was possible to achieve the release behaviour of the dyes from the PLA nanoparticles. Thus, the release data were fitting to the empirical relationship given by Ritger-Peppas equation (Eq. 4). The $n$ value on the equation is the diffusion exponent characteristic of the release mechanism, and it is given by the relation between the fraction drug release at time $t\left(M_{t} / M_{\infty}\right)$. $t$ is the release time and $k$ is the kinetic constant.

$$
\frac{M_{t}}{M_{\infty}}=k \times t^{n}
$$

To determine the value of $n$, Eq. 4 is transformed and $n$ is the slope value of the plot of $\log (\%$ release) versus $\log t$ (Eq. 5).

$$
\log (\% \text { released })=\log \left(\frac{M_{T}}{M_{\infty}}\right)=\log k+n \times \log t
$$

\section{$E x$ vivo skin penetration assay}

Abdominal porcine skin was excised from freshly slaughtered pigs at Central Carnes - Matadouro Central de Entre Douro e Minho, Lda. (Lousado, Portugal), following approved protocol by DGVDireção Geral de Veterinária. Subcutaneous fat was removed, the skin was washed with phosphate-buffered saline (PBS, pH 7.4) and placed in Franz Diffusion Cells (PermeGear Inc., Bethlehem, PA, U.S.A.) with the stratum corneum facing the donor compartment and the dermis facing the receptor compartment. After the acclimatization of the tissue to the receptor phase (PBS, pH 7.4, thermostatically maintained at $37^{\circ} \mathrm{C}$ ), the skin surface was incubated with dye-loaded nanoparticles suspensions for $24 \mathrm{~h}$. Vertical histological cuts $(20 \mu \mathrm{m}$ thickness) of frozen skin sections were made using a Leica CM1900 cryostat (Leica Microsystems, Numsloch, Germany). The distribution of nanoparticle inside the skin was investigated in histological cuts by bright field and fluorescence microscopy - Leica DM 5000B Microscope (Leica Microsystems) [4].

\section{In vitro cytotoxicity}

NCTC2544 (human skin keratinocytes) cells were cultured in $75-\mathrm{cm}^{2}$ culture flasks containing Dulbecco's modified Eagle's medium (DMEM) supplemented with $7 \%(\mathrm{v} / \mathrm{v})$ foetal bovine serum (FBS) and $1 \%(\mathrm{v} / \mathrm{v})$ of penicillin/streptomycin solution. At every 2-3 days, cell culture medium was refreshed.

To assess the skin toxicity of PLA nanoparticles, NCTC2544 cells were seeded on 24-well tissue culture polystyrene plates (TPP, Switzerland) at the initial density of $4.5 \times 10^{4}$ cells per well. The next day, fresh medium containing different volume fractions of aqueous suspensions of PLA nanoparticles was added to the cells. Before each experiment, the suspensions were sterilized with 0.45 $\mu \mathrm{m}$ filter (SARSTEDT, Nümbrecht, Germany). Several controls were performed simultaneously: the death control with 10\% (v/v) DMSO, the $100 \%$ viability control with just fresh medium and the solvent dilution controls with water instead of PLA nanoparticle suspensions at 5\%, 10\% and 20\% (v/v). Cell viability at 24, 48 and $72 \mathrm{~h}$ 
of exposure were determined using the MTT assay - a quantitative colorimetric assay where the yellow tetrazolium salt MTT is reduced by living cells to blue formazan crystals [17]. Absorbance values at 570 and $690 \mathrm{~nm}$ were measured in a Spectramax 340PC microplate reader (Molecular Devices, Sunnyvale, CA, U.S.A.). Percentages of cell viability were calculated considering the $100 \%$ cell viability control.

\section{Statistical analysis}

All the results are expressed as mean value \pm standard deviation (SD) of at least three experiments. Statistical analysis was performed with GRAPHPAD PRISM version 6.05 for Windows (GraphPad Software, La Jolla, CA, U.S.A.), using analysis of variance (ANOVA). The differences were considered statistically significant at $P<0.05$.

\section{Results and discussion}

\section{Preparation of PLA nanoparticles suitable for hair follicle targeting}

The developed nanoparticles had a net negative surface charge. It is known that PLA chains have carboxyl groups at their extremity and, when they aggregate to form nanoparticles, these groups tends to be located in the aqueous environment, creating a negative surface charge [18]. Independently of the tested processing conditions, the value of $\zeta$-potential was around $-20 \mathrm{mV}$ (data not shown).

\section{Effect of the preparation technique}

The size and size distribution of nanoparticles prepared with agitation, sonication and homogenization are shown in Fig. 1. The particles prepared with sonication were substantially smaller and more homogeneous than those produced by homogenization. This is due to the greater amount of energy supplied to the system during the sonochemical treatment. It is known that higher amounts of energy results in higher shear stress, which is inversely related to size and size distribution of particles obtained [19]. Although the size of nanoparticles obtained with sonication and agitation did not display significant differences, the use of an agitation-based protocol provided the lowest values of PDI. The dropwise addition used in this technique can induce a better spread of the organic phase into the aqueous phase, leading to precipitation of small and highly homogenous polymer aggregates in the form of nanoparticles [20]. Thus, agitation was the selected procedure for the following experiments.

\section{Effect of the addition of a non-solvent to the solvent phase}

The addition of a portion of a non-solvent to the solvent phase can be advantageous for the preparation of a smaller nanoparticles by nanoprecipitation [10]. In Fig. 2, the properties of nanoparticles prepared with acetone : water $(90: 10, \mathrm{v} / \mathrm{v})$ and acetone : ethanol ( $45: 55, \mathrm{v} / \mathrm{v})$ are compared to those obtained when only acetone was used as the solvent phase. Contrarily to expected, the use of water induced a slight increase in the mean size of nanoparticles. As PLA has low affinity to water, the polymer chains become more shrunken and overlapped, avoiding its efficient dispersion in the solvent phase. Consequently, the precipitation of larger polymer clusters can occur, and particles with larger sizes are obtained [21, 22]. The same effect can explain the increase in PDI of nanoparticles prepared with both binary mixtures tested in this study. Nevertheless, the populations obtained are still considered monodispersed. On the other hand, acetone : ethanol (45:55, v/v) promoted a decrease in the mean diameter of PLA nanoparticles. This can be explained by the high affinity of the mixture to water (acetone : ethanol is the more polar solvent phase tested). According to the literature, the higher the affinity of the solvent phase to the non-solvent phase, the faster its diffusion and particles with smaller sizes are formed [23-25]. Considering the balance between the size and size distribution of nanoparticles obtained, acetone : ethanol was chosen as the optimal solvent phase for the preparation of PLA nanoparticles.

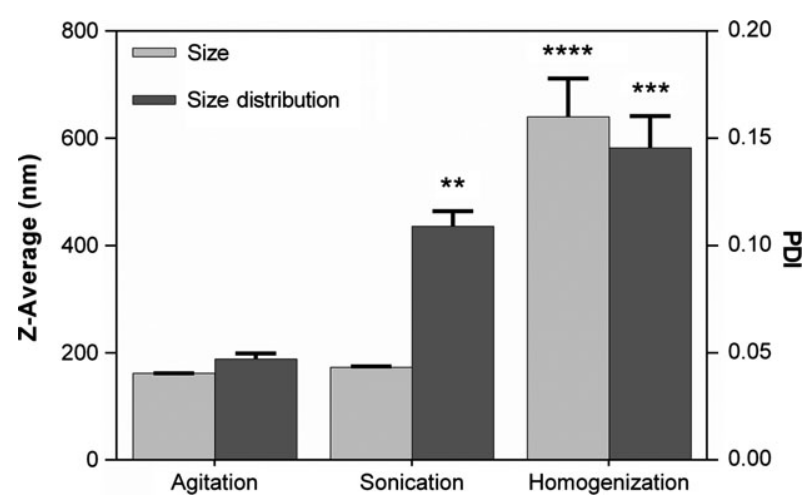

Figure 1 Size and size distribution of poly (D,L-lactide) (PLA) nanoparticles prepared by nanoprecipitation, using three different techniques to mix the solvent phase (acetone) with the aqueous phase; concentration of Pluronic ${ }^{\circledR}$ F68 in the resultant solution was $0.6 \%(\mathrm{w} / \mathrm{w})$. Data were analysed by oneway ANOVA, followed by post-hoc Dunnett's test. **P $\leq 0.01 ; * * * P \leq 0.001$ or $* * * * P \leq 0.0001$, compared to Agitation (used as reference as it provided the smallest mean size and size distribution of PLA nanoparticles).

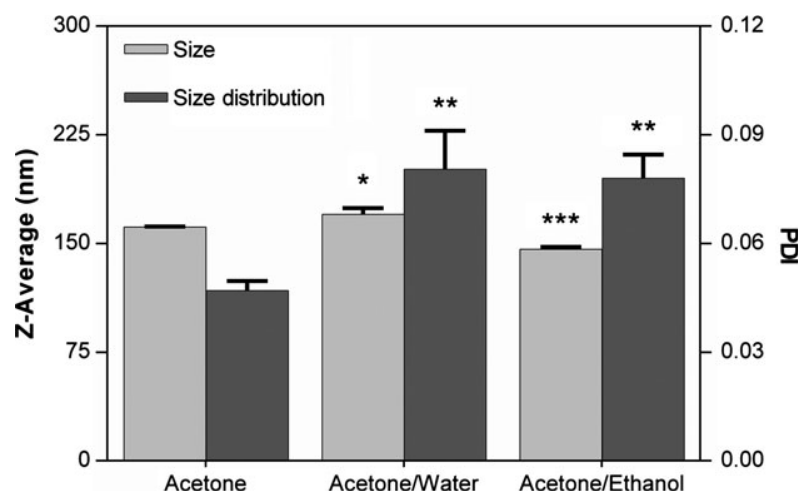

Figure 2 Size and size distribution of poly (D,L-lactide) nanoparticles prepared by nanoprecipitation, using $10 \%(\mathrm{v} / \mathrm{v})$ of water or $55 \%(\mathrm{v} / \mathrm{v})$ of ethanol in the solvent phase (acetone). Agitation was used to mix the solvent phase with the aqueous phase; concentration of Pluronic ${ }^{\circledR}$ F68 in the resultant solution was $0.6 \%(\mathrm{w} / \mathrm{w})$. Data were analysed by one-way ANOVA, followed by post-hoc Dunnett's test. $* P \leq 0.05 ; * * P \leq 0.01 ; * * * P \leq 0.001$, compared to the results obtained when only Acetone was used as the solvent phase. 


\section{Effect of the surfactant concentration}

Increasing concentrations of polymeric surfactants are responsible for an enhancement of the aqueous phase viscosity which can decrease the wavy movement, collision, aggregation (especially during the early stages of the precipitation) and further size of the particles [26]. Nevertheless, in this work, as the concentration of Pluronic ${ }^{\circledR}$ F68 increased, the mean diameter of PLA nanoparticles prepared also increased in a statistically significant manner (Fig. 3). It is possible that the increased viscosity of the aqueous phase had hindered the diffusion of the solvent phase. As mentioned before, larger nanoparticles are formed for slower solvent phase diffusion rates [23-25]. Also, it is known that Pluronic ${ }^{\circledR}$ F68 is adsorbed on the surface on nanoparticles and due to the hydration of Poly (ethylene glycol; PEG) present in its composition, the size of nanoparticles can increase. The hydrodynamic radii of PEG polymers highly hydrated (two to three water molecules per ethylene glycol unit) is approximately 5- to 10 -fold greater than would be predict by their nominal molecular weight [27]. Regarding the PDI, until $1.5 \%(\mathrm{w} / \mathrm{w})$ of surfactant, a stabilizer effect was observed. On the other hand, at $2 \%(\mathrm{w} / \mathrm{w})$, the effect of the viscosity overpowers the stabilizer effect of the surfactant, justifying the high heterogeneity of the particle populations $[15,25,28-30]$. Taking both results into consideration, $0.6 \%(\mathrm{v} / \mathrm{v})$ of Pluronic ${ }^{\circledR}$ F68 enables the preparation of the most adequate PLA nanoparticles for follicular targeting.

\section{Efficiency of nanoparticles formation}

The calculated nanoparticles yield, using $55 \%$ of ethanol in the solvent phase, was $87 \pm 7 \%$. At high percentages of ethanol, the diffusion of the organic phase into the aqueous phase is very fast and higher amounts of polymer can immediately precipitate before its agglomeration into particles occurs [23-25]. Thus, lower fractions of this solvent in the organic phase were tested to clarify its effects on the yield of PLA nanoparticles. Contrarily to what was expected, no significant increase on the yield of nanoparticles was observed (Table I). However, for volume fractions of ethanol below 55\%, the

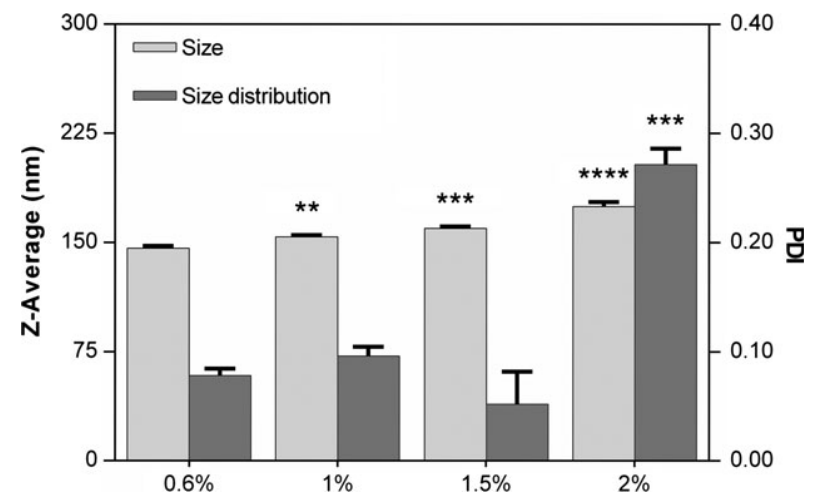

Figure 3 Size and size distribution of poly (D,L-lactide) nanoparticles prepared by nanoprecipitation, using increasing concentrations of surfactant (Pluronic ${ }^{\circledR}$ F68). Agitation was used to mix the solvent phase - acetone : ethanol $(45: 55, \mathrm{v} / \mathrm{v})$ - with the aqueous phase. Data were analysed by one-way ANOVA, followed by post-hoc Dunnett's test. $* * P \leq 0.01$; $* * * P \leq 0.001$ or $* * * * P \leq 0.0001$, compared to $0.6 \%$ (concentration used in the previous optimizations).
Table I Effect of decreasing volume fractions of ethanol (used in the solvent phase) on the yield and properties of poly (D,L-lactide) nanoparticles

\begin{tabular}{lllll}
\hline $\begin{array}{l}\text { Volume } \\
\text { fraction of } \\
\text { ethanol (\%) }\end{array}$ & $\begin{array}{l}\text { Yield of } \\
\text { nanoparticles } \\
(\%)\end{array}$ & $\begin{array}{l}\text { Z-Average } \\
(\mathbf{n m})\end{array}$ & PDI & $\begin{array}{l}\text { Zeta-potential } \\
(\mathbf{m V})\end{array}$ \\
\hline & & & & \\
\hline 55 & $87 \pm 7$ & $146 \pm 2$ & $0.078 \pm 0.007$ & $-21.5 \pm 1.3$ \\
50 & $91 \pm 1$ & $151 \pm 1^{\star}$ & $0.074 \pm 0.011$ & $-18.1 \pm 0.5^{\star}$ \\
45 & $93 \pm 2$ & $138 \pm 1^{\star \star}$ & $0.074 \pm 0.010$ & $-15.3 \pm 0.9^{\star \star}$ \\
\hline
\end{tabular}

These particles were prepared by nanoprecipitation, using agitation to mix the solvent phase and the aqueous phase; concentration of Pluronic ${ }^{\circledR}$ F68 in the resultant solution was $0.6 \%(\mathrm{w} / \mathrm{w})$. Data were analysed by one-way ANOVA, followed by post-hoc Dunnett's test. $* P \leq 0.05$ and $* * P \leq 0.01$, compared to the maximal tested volume fraction of ethanol $(55 \%)$.

method seems to be more reproducible as lower standard deviations were obtained.

The properties of nanoparticles, prepared with different volume fractions of ethanol in the solvent phase, were also compared (Table I). Despite the PDI remained unchanged, alterations on the size and $\zeta$-potential were registered. For $45 \%(\mathrm{v} / \mathrm{v})$, there was a considerable decrease on the mean diameter and $\zeta$-potential (absolute value) of nanoparticles when compared to the values obtained at other ethanol percentages. The value of surface charge obtained can be problematic during the storage of nanoparticles because it will not be negative enough to prevent their aggregation by electrostatic repulsions [31]. Regarding to $50 \%(\mathrm{v} / \mathrm{v})$ of ethanol, a slight increase in the mean size and surface charge of nanoparticles was noticed, but the use of these particles for hair follicle targeting is not impaired. Consequently, $50 \%(\mathrm{v} / \mathrm{v})$ was chosen as the optimal volume fraction of ethanol in the solvent phase: it constitutes the best compromise between yield of nanoparticles, reproducibility of the method and suitability of nanoparticles properties when follicular targeting is intended.

\section{Morphology and physical stability of nanoparticles}

The morphology of PLA nanoparticles obtained with the optimal conditions stated before can be observed in Fig. 4(A). The shape of these particles is spherical. This morphology is optimal for a drug delivery system, as it provides the longest diffusion pathways and protection of incorporated drugs due to the minimum contact with the aqueous environment [26]. Moreover, this suspension proved to be physically stable after 6 months of storage at $4^{\circ} \mathrm{C}$ as no significant variations in size, PDI and $\zeta$-potential (data not shown) were noticed (Fig. 4B).

\section{Entrapment efficiency of model compounds}

The dye-loaded PLA nanoparticles did not show significant differences on mean diameter, PDI and $\zeta$-potential when compared to the empty nanoparticles. Also, no significant changes were noticed between dye-loaded nanoparticles formulations and empty nanoparticles formulations, regarding the efficiency of nanoparticles formation (data not shown).

The encapsulation of both dyes proved to be highly efficient, and the results obtained are shown in Table II. Although a high entrapment efficiency was expected for NR (a lipophilic compound), 
A
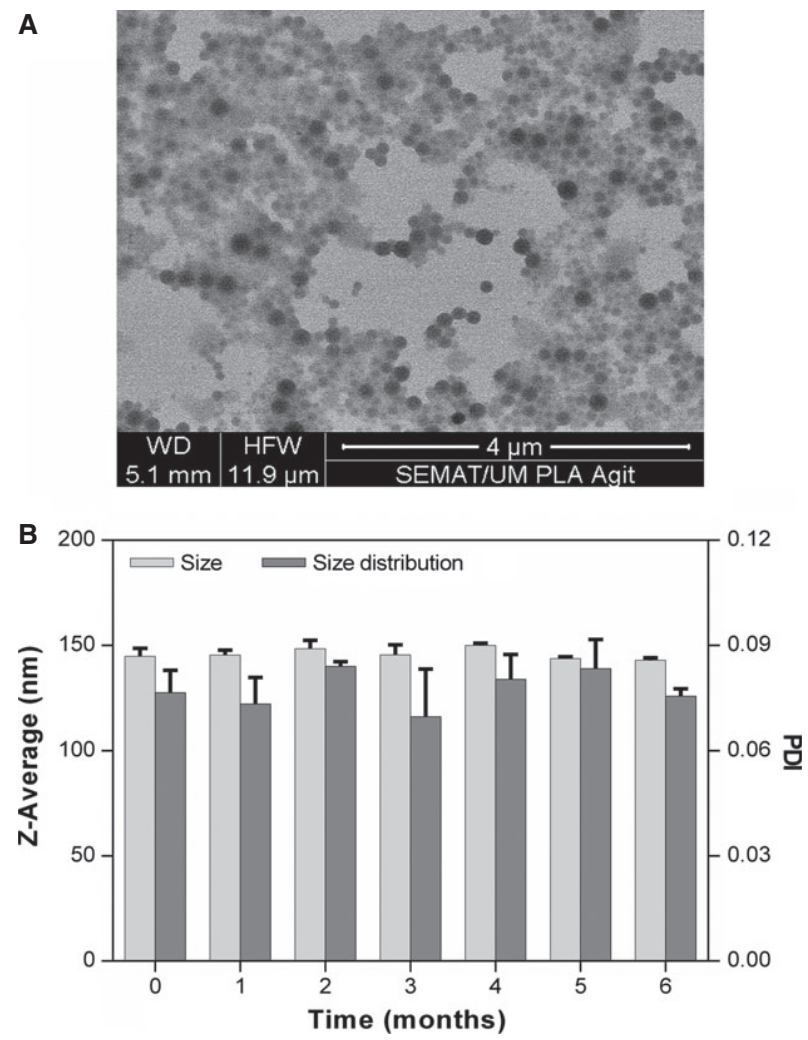

Figure 4 STEM photograph $(\times 25000$ magnification $)(A)$ and stability over 6 months (B) of poly (D,L-lactide) nanoparticles. These particles were prepared by nanoprecipitation, using agitation to mix the solvent phase acetone : ethanol $(50: 50, \mathrm{v} / \mathrm{v})$ - and the aqueous phase; concentration of Pluronic ${ }^{\circledR} \mathrm{F} 68$ in the resultant solution was $0.6 \%(\mathrm{w} / \mathrm{w})$. Data were analysed by one-way ANOVA, followed by post-hoc Dunnett's test. No statistical significant differences were found, compared to time point 0 months.

Table II Effect of the compounds nature (lipophilic or hydrophilic) on the entrapment and loading efficiencies into poly (D,L-lactide) nanoparticles

\begin{tabular}{lll}
\hline $\begin{array}{l}\text { Entrapped } \\
\text { compound }\end{array}$ & $\begin{array}{l}\text { Entrapment } \\
\text { efficiency (\%) }\end{array}$ & $\begin{array}{l}\text { Loading } \\
\text { efficiency (\%) }\end{array}$ \\
\hline Nile red & $89 \pm 7$ & $0.0488 \pm 0.0019$ \\
FITC & $83 \pm 2$ & $0.0508 \pm 0.0004$ \\
\hline
\end{tabular}

These particles were prepared by nanoprecipitation, dissolving $1 \%(\mathrm{w} / \mathrm{v})$ of Nile Red or fluorescein 5(6)-isothiocyanate (FITC) in the solvent phase - acetone : ethanol $(50: 50, v / v)$. Agitation was used to mix solvent phase with the aqueous phase, and the concentration of Pluronic ${ }^{\circledR}$ F68 in the resultant solution was $0.6 \%(w / w)$.

the results obtained for FITC are surprising taking into account that usually, nanoprecipitation is ineffective to encapsulate compounds of hydrophilic nature. Due to their water-soluble nature, they rapid partioning from the core of nanoparticles into the aqueous phase, leading to considerable decrease in their encapsulation efficiency [22]. However, the composition of the solvent phase used

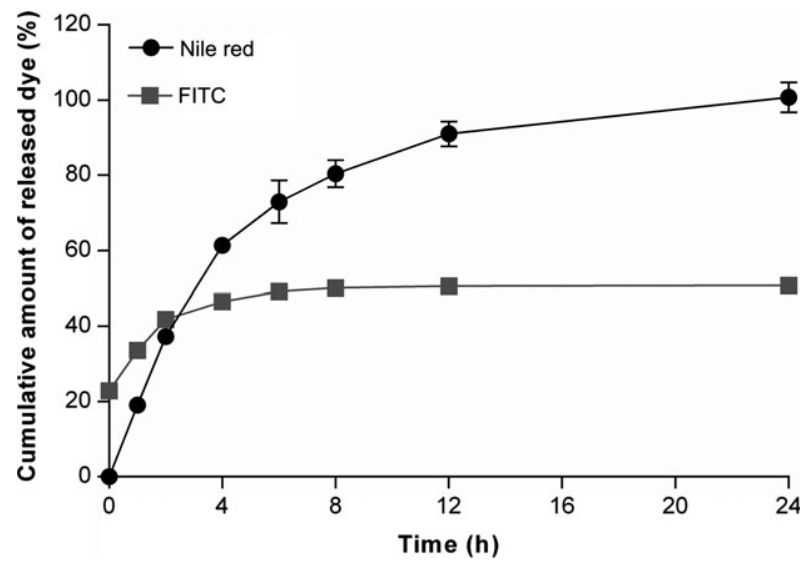

Figure 5 In vitro release profile of nile red and fluorescein 5(6)-isothiocyanate (FITC) from poly (D,L-lactide) nanoparticles. These particles were prepared by nanoprecipitation, using agitation to mix the solvent phase acetone : ethanol $(50: 50, \mathrm{v} / \mathrm{v})$ - with the aqueous phase; the concentration of Pluronic ${ }^{\circledR}$ F68 in the resultant solution was $0.6 \%(\mathrm{w} / \mathrm{w})$. The initial concentration of dyes in the solvent phase was $1 \%(\mathrm{w} / \mathrm{v})$.

makes its diffusion rate and consequently solidification of the nanoparticles very fast. Consequently, that leakage of large amounts of FITC is avoided. The increased viscosity of the organic phase also helps to increase the diffusional resistance of FITC, and it was previously involved on the achievement of high encapsulation efficiency of some other molecules [19].

In addition to the entrapment efficiency, the loading efficiency was also determined for both dyes (Table II). As formulations have similar yields of nanoparticles and entrapment efficiencies, it is not surprising that no significant differences were found for loading efficiencies obtained for NR-loaded and FITC-loaded nanoparticles.

\section{Release profile of dyes from PLA nanoparticles}

In vitro release of NR and FITC was evaluated using artificial sebum (IPM) (Fig. 5). Both kinetic profiles exhibit a typical biphasic release phenomenon. The initial burst release is attributed to the fraction of the dyes which is adsorbed/weakly bound to the large surface area of nanoparticles or entrapped in the matrix near the surface of particles. The following slow and constant release over the remaining time is due to the diffusion of compounds and erosion of the polymeric matrix [32].

When particles were placed in contact with IPM $(0 \mathrm{~h})$, higher amounts of FITC were immediately released from nanoparticles. As mentioned before, during the formation of nanoparticles, FITC have a tendency to rapidly partioning into the aqueous phase (due to its hydrophilic nature). Despite this phenomenon usually lead to low entrapment efficiencies, in this study, that did not happen. However, it is possible that high amounts of FITC have been poorly entrapped in the matrix near to the nanoparticles surface. Upon contact with IPM, these FITC molecules are immediately released due to the destabilization of nanoparticles [31, 33].

After the first time point, NR showed a faster release from PLA nanoparticles: $100 \%$ was completely achieved within $24 \mathrm{~h}$. This is tightly coupled to its lipophilic nature. Upon contact with 
IPM, a destabilization of the particles occurs (due to the dissolution of the polymer in the lipophilic phase), allowing the solvent to penetrate into their cores. As lipophilic compounds have high affinity to IPM, their partioning between the core of nanoparticles and the lipophilic solvent occurs easily, leading to their rapidly diffusion out of the particles [34]. This rapid leakage contributes to destabilize even more the particles, leading to a loss of their particulate state, which promotes a further increase in the release of the entrapped lipophilic compound [35]. The

Table III Dye release kinetic data obtained from fitting experimental release of Nile Red and fluorescein 5(6)-isothiocyanate (FITC) from poly (D,L-lactide) nanoparticles to Ritger-Peppas modified equation, where $n$ is the diffusion exponent and $R^{2}$ is the correlation coefficient

\begin{tabular}{lll}
\hline Released compound & $\boldsymbol{n}$ & $\boldsymbol{R}^{2}$ \\
\hline Nile red & & \\
FITC & 0.847 & 0.993 \\
& 0.209 & 0.959 \\
\hline
\end{tabular}

fast in vitro release of NR is of great interest in the particular case of hair follicle targeting because it is possible to assume that in vivo, the full release of lipophilic drugs in the hair follicle canal will occur before the excretion of nanoparticles due to sebum production (8 days) [36]. On the other hand, as hydrophilic compounds (like FITC) have poor solubility in the lipophilic solvent, they partioning into IPM at a slower rate and their diffusion outside the particles is hindered, resulting in prolonged release kinetics [35, 37]

To confirm the mechanism of drug release stated above for NR (diffusion and polymeric matrix degradation) and FITC (mainly diffusion), the empirical expression proposed by RitgerPeppas (based on the Fickian diffusion equation) was used. For a sphere, a diffusion of first order is observed when has the limiting value of 0.43 . Between 0.43 and 0.85 , anomalous transport is observed coupling Fickian diffusion and polymer degradation. Finally, for $n=0.85$, Case II transport (polymer relaxation/degradation) occurs, leading to a zero-order release [26]. For PLA nanoparticles produced in this work, the $n$ value obtained for the tested dyes are shown in Table III. According to the values, anomalous transport (diffusion and polymer degradation) and Fickian diffusion of first order govern the release of Nile Red
A

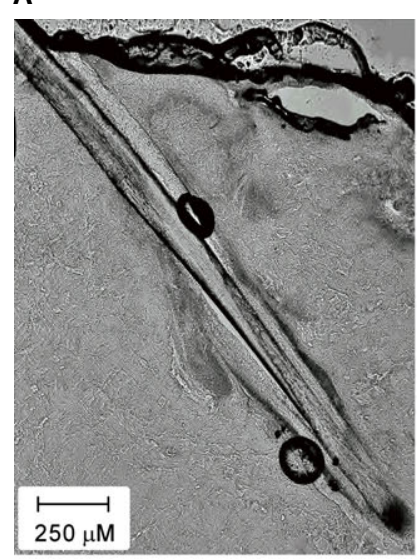

B
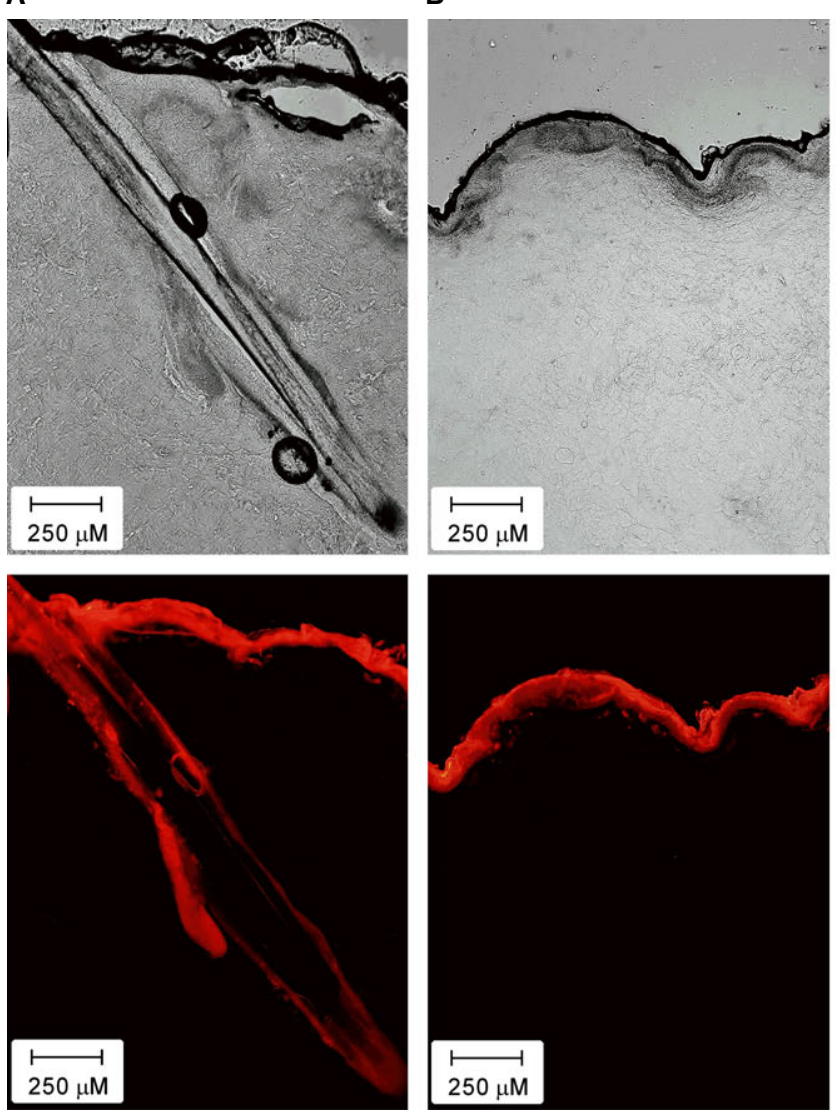

C
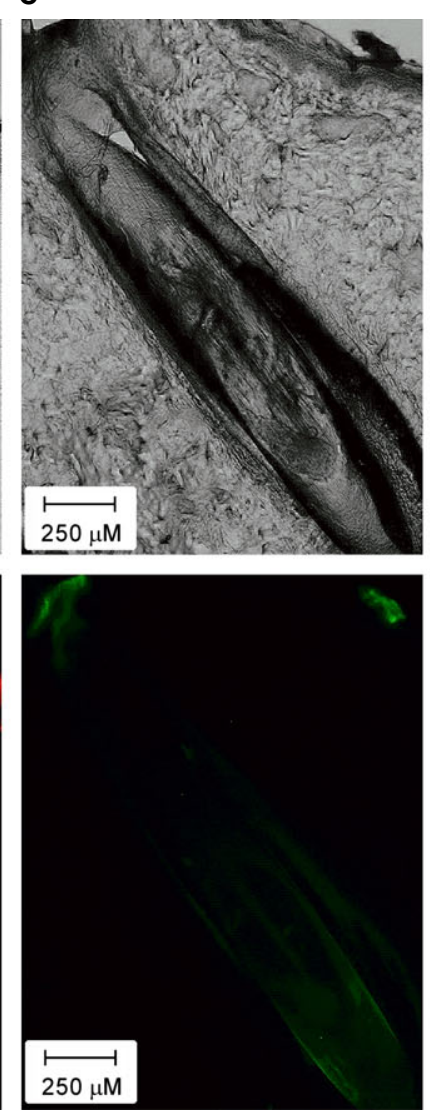

D
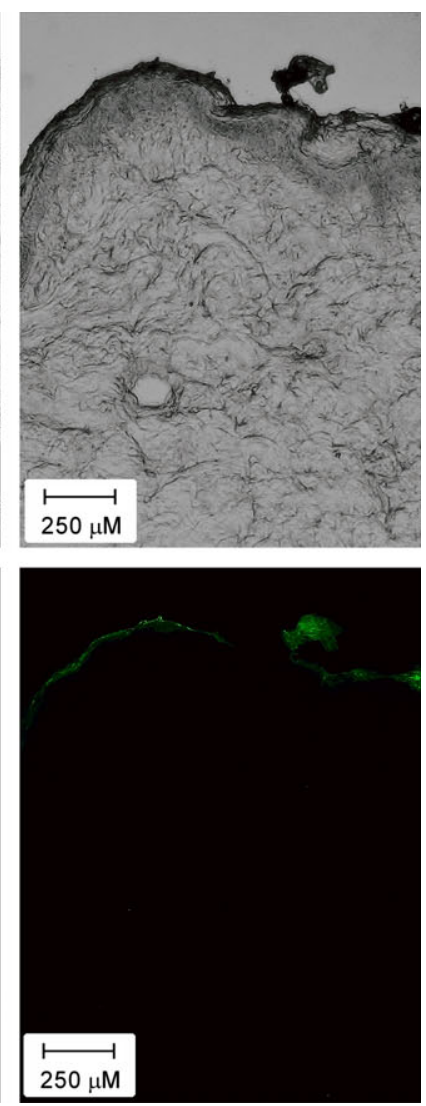

Figure 6 Bright field (top) and fluorescence (bottom) images of hair follicles (A,C) and stratum corneum (B,D) of porcine skin cryosections, after 24 h of incubation with nile red-loaded (A,B) and fluorescein $5(6)$-isothiocyanate-loaded (C,D) poly (D,L-lactide) nanoparticles $(\times 5$ magnification). These particles were prepared by nanoprecipitation, using agitation to mix the solvent phase - acetone : ethanol (50:50, v/v) - with the aqueous phase; the concentration of Pluronic ${ }^{\circledR}$ F 68 in the resultant solution was $0.6 \%(\mathrm{w} / \mathrm{w})$. The initial concentration of dyes in the solvent phase was $1 \%(\mathrm{w} / \mathrm{v})$. 


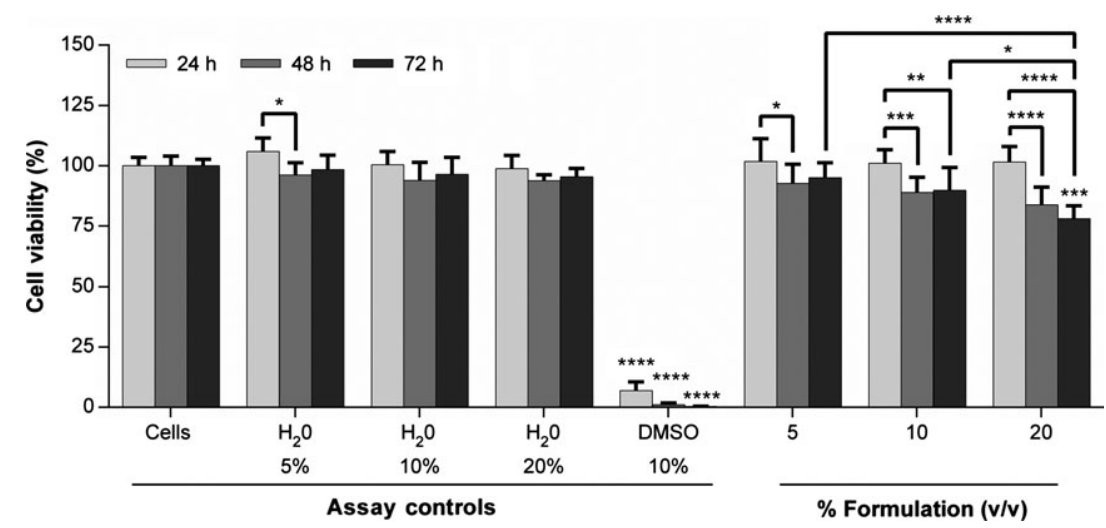

Figure 7 NCTC2544 cell viability (MTT assay) was determined at 24, 48 and $72 \mathrm{~h}$ of exposure to different volume fractions of poly (D,L-lactide) nanoparticles suspension. These particles were prepared by nanoprecipitation, using agitation to mix the solvent phase - acetone : ethanol (50:50, v/v) - and the aqueous phase; concentration of Pluronic ${ }^{\circledR}$ F68 in the resultant solution was $0.6 \%(\mathrm{w} / \mathrm{w})$. Values for tested samples are presented in relation to the cells control (cells cultured with medium scored for $100 \%$ of viability). Data were analysed by two-way ANOVA, followed by post-hoc Tukey's test. $* P \leq 0.05 ; * * P \leq 0.01$; *** $P \leq 0.001$ or $* * * * P \leq 0.0001$, when conditions were compared with the corresponding medium dilution control or among each other.

(lipophilic) and FITC (hydrophilic) from PLA nanoparticles, respectively.

\section{Penetration of nanoparticles into the hair follicles after topical application}

The penetration profile of PLA nanoparticles was assessed on porcine skin which resembles human skin regarding its permeability properties [38]. NR-loaded and FITC-loaded nanoparticles were observed inside the hair follicles along the entire follicular duct (Fig. 6A,C), reaching the hair bulb (where melanocytes can be found) [3]. Although some nanoparticles have been trapped in the stratum corne$u m$, they did not reach the viable epidermis as no fluorescence was detected in this skin layer (Fig. 6B,D). These findings confirm that the obtained PLA nanoparticles efficiently and selectively target the hair follicles after topical application, even without the use of massage. Massage is used in ex vivo studies to simulate the hair movement that occurs physiologically under in vivo conditions and facilitate the entrance of nanoparticles into the hair follicles [39].

\section{Cytotoxicity of nanoparticles using a reference skin cell line}

The cytotoxicity of PLA nanoparticles was assessed in in vitro cultured keratinocytes, as a measure of the skin toxicity. NCTC2544 cells were exposed to different concentrations of nanoparticles ranging from 165 to $660 \mu \mathrm{g} \mathrm{mL}^{-1}$ [5-20\% (v/v) of the optimal formulation], and cell viability measured at 24,48 and $72 \mathrm{~h}$. According to the results (Fig. 7), none of the tested amounts of PLA nanoparticles had a biological significant toxicity (cell viability $>78 \%$ ). For an exposure period of $24 \mathrm{~h}$, there was no decrease in keratinocytes viability when compared to the medium dilution control. For longer periods of exposure, besides the medium dilution effect (statistically significant just for a $72-\mathrm{h}$ exposure to $20 \%(\mathrm{v} / \mathrm{v}$ ) of formulation), a decrease in viability was observed for concentrations higher than $165 \mu \mathrm{g} \mathrm{mL}^{-1}(10-20 \%)$, with varied degrees of statistical significance. Despite this, the range of nanoparticles concentrations tested can be safely applied to the skin.

\section{Conclusions}

On this study, an optimized nanoprecipitation method is described. PLA nanoparticles prepared are well suited to penetrate into the hair follicles duct, reaching the hair bulb without reaching the viable epidermis. This site-specific target of hair follicles has great impact in hair follicle therapies. The high yield obtained, the stability over 6 months, the absence of toxicity in a skin cell line model, the efficient entrapment and suitable in vitro release of lipophilic and hydrophilic model compounds make these formulations viable for industrial purposes when the development of new cosmetics for human hair is intended.

\section{Acknowledgements}

The authors thank to the FCT Strategic Project PEst-OE/EQB/ LA0023/2013 and to the Project "BioHealth - Biotechnology and Bioengineering approaches to improve health quality”, Ref. NORTE07-0124-FEDER-000027, co-funded by the Programa Operacional Regional do Norte (ON.2 - O Novo Norte), QREN, FEDER. This work was partly supported by FEDER through POFC-582 COMPETE and by Portuguese funds from FCT- Fundação Para a Ciência e a Tecnologia through the project PEst-OE/BIA/UI4050/2014. The authors thank C. Botelho for technical assistance.

\section{References}

1. Morganti, P. Use and potential of nanotechnology in cosmetic dermatology. Clin. Cosmet. Investig. Dermatol. 3, 5-13 (2010).

2. Vogt, A., Mandt, N., Lademann, J., Schaefer, H. and Blume-Peytavi, U. Follicular target- ing - a promising tool in selective dermatotherapy. J. Invest. Dermatol. 10, 252-255 (2005).

3. Papakostas, D., Rancan, F., Sterry, W., Blume-Peytavi, U. and Vogt, A. Nanoparti- cles in dermatology. Arch. Dermatol. Res. 303, 533-550 (2011).

4. Rancan, F., Papakostas, D., Hadam, S. et al. Investigation of polylactic acid (PLA) nanoparticles as drug delivery systems for 
local dermatotherapy. Pharm. Res. 26, 2027-2036 (2009).

5. Meidan, V., Bonner, M. and Michniak, B. Transfollicular drug delivery - is it a reality? Int. J. Pharm. 306, 1-14 (2005).

6. Araújo, R., Fernandes, M., Cavaco-Paulo, A. and Gomes, A. Biology of human hair: know your hair to control It. Adv. Biochem. Eng. Biotechnol. 125, 121-143 (2011).

7. Hughes, G.A. Nanostructure-mediated drug delivery. Nanomedicine 1, 22-30 (2005).

8. Prow, T.W., Grice, J.E., Lin, L.L., Faye, R., Butler, M., Becker, W. et al. Nanoparticles and microparticles for skin drug delivery. Adv. Drug. Deliv. Rev. 63, 470-491 (2011).

9. Guterres, S.S., Alves, M.P. and Pohlmann, A.R. Polymeric nanoparticles, nanospheres and nanocapsules, for cutaneous applications. Drug Target Insights 2, 147-157 (2007).

10. Fessi, H., Devissaguet, J.P., Puisieux, F. and Thies, C.. Process for the preparation of dispersible colloidal systems of a substance in the form of nanoparticles. US (1991).

11. Wosicka, H. and Cal, K. Targeting to the hair follicles: current status and potential. J. Dermatol. Sci 57, 83-89 (2010).

12. Legrand, P., Lesieur, S., Bochot, A., Gref, R., Raatjes, W., Barratt, G., Vauthier, C. Influence of polymer behaviour in organic solution on the production of polylactide nanoparticles by nanoprecipitation. Int. J. Pharm. 344, 33-43 (2007).

13. Liu, M., Dong, J., Yang, Y., Yang, X. and $\mathrm{Xu}, \mathrm{H}$. Characterization and release of triptolide-loaded poly (D, L -lactic acid) nanoparticles. Eur. Polym. J. 41, 375-382 (2005).

14. Silva, R., Ferreira, H., Little, C. and CavacoPaulo, A.. Effect of ultrasound parameters for unilamellar liposome preparation. Ultrason. Sonochem. 17, 628-632 (2010).

15. Alvarez-Román, R., Naik, A., Kalia, Y.N., Guy, R.H. and Fessi, H. Enhancement of topical delivery from biodegradable nanoparticles. Pharm. Res. 21, 1818-1825 (2004).

16. Gaucher, V.E. and Poreba, M. Poly (N-vinylpyrrolidone) - block-poly (D,L-lactide) as polymeric emulsifier for the preparation of biodegradable nanoparticles. J. Pharm. Sci. 96, 1763-1775 (2007).

17. Van Meerloo, J., Kaspers, G.J.L. and Cloos, J. Cell sensitivity assays: the MTT assay. Methods Mol. Biol. 731, 237-245 (2011).
18. Santander-Ortea, M.J., Jódar-Reyes, A.B., Csaba, N., Bastos-González, D. and OrtegaVinuesa, J.L. Colloidal stability of Pluronic F68-coated PLGA nanoparticles: a variety of stabilisation mechanisms. J. Colloid Interface Sci. 302, 522-529 (2006).

19. Moghimi, H., Williams, A. and Barry, B. A lamellar matrix model for stratum corneum intercellular lipids. II. Effect of geometry of the stratum corneum on permeation of model drugs 5-fluorouracil and oestradiol. Int. J. Pharm. 131, 117-129 (1996).

20. Schubert, S., Delaney, J.T. and Schubert, U.S. Nanoprecipitation and nanoformulation of polymers: from history to powerful possibilities beyond poly (lactic acid). Soft Matter 7, 1581-1588 (2011).

21. Bilati, U., All, E. and Doelker, E. Development of a nanoprecipitation method intended for the entrapment of hydrophilic drugs into nanoparticles. Eur. J. Pharm. Sci. 24, 67-75 (2005).

22. Donnell, P.B.O. and Mcginity, J.W. Preparation of microspheres by the solvent evaporation technique. Adv. Drug Deliv. Rev. 28, 25-42 (1997).

23. Wischke, C. and Schwendeman, S.P. Principles of encapsulating hydrophobic drugs in PLA/PLGA microparticles. Int. J. Pharm. 364, 298-327 (2008).

24. Peltonen, L., Koistinen, P., Karjalainen, M., Hakkien, A. and Hirvonen, J. The effect of cosolvents on the formulation of nanoparticles from low-molecular-weight poly (L) Lactide. AAPS PharmSciTech. 3, 1-7 (2002).

25. Guhagarkar, S.A., Malshe, V.C. and Devarajan, P.V. Nanoparticles of polyethylene sebacate: a new biodegradable polymer. AAPS PharmSciTech. 10, 935-942 (2009).

26. Silva, R., Ferreira, H., Carvalho, A.C., Gomes, A.C. and Cavaco-paulo, A. Protein microspheres as suitable devices for piroxicam release. Colloids Surf. B Biointerfaces 92 , 277-285 (2012).

27. Harris, J.M. and Chess, R.B. Effect of pegylation on pharmaceuticals. Nat. Rev. Drug Discov. 2, 214-221 (2003).

28. Mao, S., Xu, J., Cai, C., Germershaus, O., Schaper, A. and Kissel, T. Effect of WOW process parameters on morphology and burst release of FITC-dextran loaded PLGA microspheres. Int. J. Pharm. 334, 137-148 (2007).

29. Vandervoort, J. and Ludwig, A. Preparation factors affecting the properties of polylactide nanoparticles: a factorial design study. Pharmazie. 56, 484-488 (2001).

30. Zweers, M.L.T., Grijpma, D.W., Engbers, G.H.M. and Feijen, J. The preparation of monodisperse biodegradable polyester nanoparticles with a controlled size. J. Biomed. Mater. Res. Part B Appl. Biomater. 66B, 559-566 (2002).

31. Hans, M.L. and Lowman, A.M. Biodegradable nanoparticles for drug delivery and targeting. Curr. Opin. Solid State Mater. Sci. 6, 319-327 (2002).

32. Soppimath, K.S., Aminabhavi, T.M., Kulkarni, A.R. and Rudzinski, W.E. Biodegradable polymeric nanoparticles as drug delivery devices. J. Control. Release 70, 120 (2001).

33. Chawla, J.S. and Amiji, M.M. Biodegradable poly (o-caprolactone) nanoparticles for tumor-targeted delivery of tamoxifen. Int. J. Pharm. 249, 127-138 (2002).

34. Jung, S., Otberg, N., Thiede, G., Richter, H., Sterry, W., Panzner, S., Lademann, J. Innovative liposomes as a transfollicular drug delivery system: penetration into porcine hair follicles. J. Invest. Dermatol. 126, 2-6 (2006).

35. Rancan, F., Todorova, A., Hadam, S. et al. Stability of polylactic acid particles and release of fluorochromes upon topical application on human skin explants. Eur. J. Pharm. Biopharm. 80, 76-84 (2012).

36. Rolland, A., Wagner, N., Chatelus, A., Shroot, B. and Schaefer, H. Site-specific drug delivery to pilosebaceous structures using polymeric microspheres. Pharm. Res. 10, 1738-1744 (1993).

37. Leo, E., Brina, B., Forni, F. and Vandelli, M.A. In vitro evaluation of PLA nanoparticles containing a lipophilic drug in watersoluble or insoluble form. Int. J. Pharm. 278, 133-141 (2004).

38. Hammond, S.A., Tsonis, C., Sellins, K., Rushlow, K., Scharton-Kersten, T., Colditz, L., Glenn, G.M. Transcutaneous immunization of domestic animals: opportunities and challenges. Adv. Drug Deliv. Rev. 43, 45-55 (2000).

39. Lademann, J., Richter, H., Schanzer, S., Knorr, F., Meinke, M., Sterry, W., Patzelt, A. Penetration and storage of particles in human skin: perspectives and safety aspects. Eur. J. Pharm. Biopharm. 77, 465468 (2011). 